\title{
Extracellular Vesicles, Apoptotic Bodies and Mitochondria: Stem Cell Bioproducts for Organ Regeneration
}

\author{
Natalia Gebara ${ }^{1} \cdot$ Andrea Rossi $^{1} \cdot$ Renata Skovronova $^{1} \cdot$ Justine Mariam Aziz ${ }^{2} \cdot$ Amish Asthana $^{2}$. \\ Benedetta Bussolati ${ }^{1,3}$ (ID
}

Published online: 27 April 2020

(C) The Author(s) 2020

\begin{abstract}
Purpose of Review In the current work, we will present the characterization of the main different stem cell-derived vesicular bioproducts with potential application in organ regeneration.

Recent Findings The therapeutic effects of stem cell therapy in organ repair, specifically those utilizing mesenchymal stromal cells, are largely dependent on the cells' release of different bio-products. Among these bio-products, extracellular vesicles (EVs) appear to play a major role due to their ability to carry and deliver bioactive material for modulation of cellular pathways in recipient cells. Concurrently, mitochondria transfer emerged as a new mechanism of cell communication, in which the bioenergetics of a damaged cell are restored. Finally, apoptotic bodies released by dying apoptotic stem cells contribute to stimulation of the tissue's stem cells and modulation of the immune response.

Summary Exploitation of isolated extracellular vesicles, mitochondria and apoptotic bodies in preclinical models of organ damage shows promising results. Here, we describe the results of the pre-clinical applications of stem cell vesicular products, as well as the first clinical trials approaching artificial administration of extracellular vesicles and mitochondria in human subjects and their possible benefits and limitations.
\end{abstract}

Keywords MSC $\cdot$ Regenerative medicine $\cdot$ MicroRNA $\cdot$ Exosomes $\cdot$ Microvesicles $\cdot$ Mitochondrial transfer $\cdot$ Apoptosis

\section{Introduction}

Organ failure is the most frequent cause of morbidity and mortality recorded in Europe and in the United States in recent decades. Organ dysfunction can be attributed to fibrosis, a pathological feature of many chronic inflammatory diseases, as its extensive remodelling of tissues leads to functional insufficiency [1]. The burden associated with fibrosis is disconcerting, representing in the United States almost half, and in

Natalia Gebara, Andrea Rossi and Renata Skovronova contributed equally to this work.

This article is part of the Topical Collection on Cellular Transplants

Benedetta Bussolati

benedetta.bussolati@unito.it

1 Department of Molecular Biotechnology and Health Sciences, University of Torino, Torino, Italy

2 Wake Forest Univ. School of Medicine, Winston-Salem, NC, USA

3 Molecular Biotechnology Centre, University of Torino, via Nizza 52, 10126 Torino, Italy the industrialized world about $30 \%$, of all deaths attributed to fibrotic heart, lung, kidney and liver diseases $[1,2]$. In addition, episodes of acute tissue injury, especially if severe and repeated, are closely associated to development of chronic organ disease [3].

It has therefore become of increasing interest in Regenerative Medicine to limit the progression of fibrosis, promote restoration of organ function in chronic settings and support organ repair after acute injury to regain tissue integrity. In this context, increasing studies underline the role of stem cell bio-products, including secreted soluble factors and extracellular vesicles (EVs), as powerful instruments in organ regeneration. EVs, in particular, have been proposed as a new form of intracellular messaging through their ability to reach distant organs and deliver the active cargo necessary for reprogramming of the target cells. In addition, EVs released by apoptotic cells, including apoptotic bodies (ApoBDs), are recently emerging as part of the therapeutic and immune-modulating mechanisms of injected stem cells within injured tissues [4] (Fig. 1).

Finally, stem cell therapy involves the transfer of mitochondria, the organelles responsible for cellular energy production, from stem cells to damaged cells. Mesenchymal stromal cells 
Fig. 1 Presentation of apoptotic and healthy cell secretome
Apoptotic cell
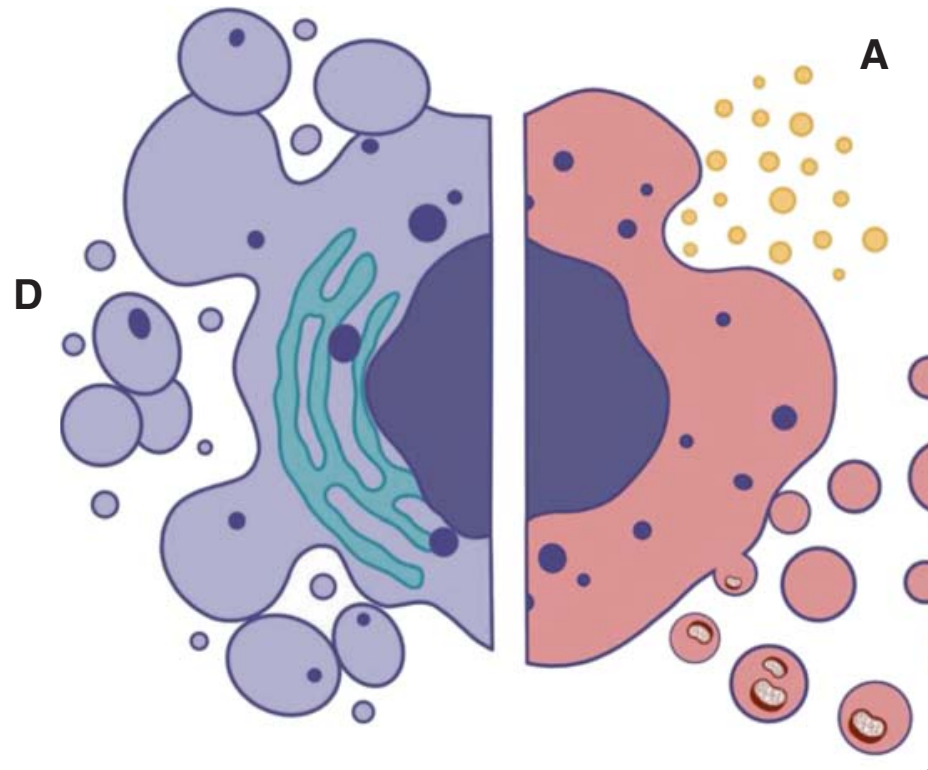

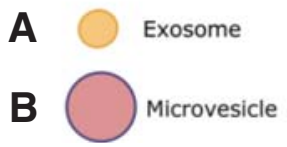

C

Healthy cell

\section{A}

B

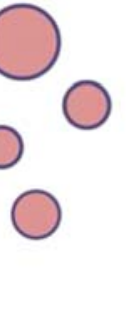

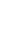

(MSCs) are shown to transfer mitochondria to the recipient cells in different ways: encapsulated within EVs [5]; via cellto-cell direct communication through tunnelling nanotubes; or through direct release of "naked" mitochondria into the extracellular microenvironment [6]. The organelle incorporates into the endogenous mitochondrial network of the damaged recipient cell that needs to be rescued, restoring its bioenergetic profile and health [7].

In this review, we will present the recent knowledge on mechanisms of action involved in the therapeutic effects of healthy and apoptotic EVs, as well as of mitochondria transfer, and the exploitation of these bio-products in preclinical models of organ damage. Finally, we will describe the first clinical trials approaching their use on human subjects and the possible benefits and limitations.

\section{Extracellular Vesicles}

Since the 'discovery' of EVs in blood plasma in 1946 by Erwin Chargaff and Rudolph West [8], interest in those cells-to-cell communicators has risen in almost all fields of biology and chemistry. EVs have been proven to naturally occur in prokaryotes, eukaryotes, plants and cells. EVs are membrane-bound, spherical particles enclosed in a lipid bilayer. In biological samples, EVs originate from their parental cell, taking up their internal and external composition. Guidelines from the International Society for Extracellular Vesicles (ISEV) classify three main categories of EVs; exosomes also named small EVs $(\sim 30-250 \mathrm{~nm})$, large EVs or microvesicles $(\sim 100-$ $1000 \mathrm{~nm})$ and ApoBDs $(>1 \mu \mathrm{m})$ [9••]. Small EVs and microvesicles are released from metabolically active cells, whereas ApoBDs are exclusively produced during cell apoptosis [10] (Fig. 1).

The content of an EV is dependent on its origin, size and the route of biogenesis. EV surface markers and cargo are specific to the three types of vesicles (Table 1) and are most commonly associated with the route of vesicle formation. The process of exosome formation begins with inward budding of early endosomes and formation of intraluminal vesicles; this involves the ESCORT complex, ALIX and tumour susceptibility gene 101 (TSG101), all of which are responsible for cargo sorting. Intraluminal vesicles mature into multivesicular bodies, followed by fusion with the cell membrane and release of vesicles into the extracellular environment. Alternatively, multi-vesicular bodies are degraded by lysosomes and their components recycled. Exosomes are distinguished by the presence of all three tetraspanin markers responsible for induction of membrane curvature (CD9, CD63 and CD81). Proteins that can be detected and are involved in exosome biogenesis include Rab, GTPases, annexin, flotillin, 
Table 1 Composition, size and biogenesis route of EVs

\begin{tabular}{|c|c|c|c|c|}
\hline Vesicle type & Origin & Size & Markers & Components \\
\hline Exosomes & $\begin{array}{l}\text { Endolysosomal pathway; } \\
\text { fusion of MVB with cell } \\
\text { membrane }\end{array}$ & $\begin{array}{c}30-250 \\
\mathrm{~nm}\end{array}$ & $\begin{array}{l}\text { CD9, CD63, CD81 ESCORT } \\
\text { components, TSG101, flotillin, } \\
\text { Annexin }\end{array}$ & $\begin{array}{l}\text { mRNA, miRNA and other non-coding RNAs; } \\
\text { membrane and cytoplasmic proteins, lipids, } \\
\text { receptors }\end{array}$ \\
\hline Microvesicles & $\begin{array}{l}\text { Cell membrane; bud off directly } \\
\text { from cell surface }\end{array}$ & $\begin{array}{l}100-1000 \\
\mathrm{~nm}\end{array}$ & $\mathrm{CD} 40$ & $\begin{array}{l}\text { mRNA, miRNA and other non-coding RNAs; } \\
\text { membrane and cytoplasmic proteins, lipids, } \\
\text { receptors }\end{array}$ \\
\hline $\begin{array}{l}\text { Apoptotic } \\
\text { bodies }\end{array}$ & $\begin{array}{l}\text { Cell Membrane; membrane } \\
\text { blebbing during apoptosis }\end{array}$ & $>1 \mu \mathrm{m}$ & $\begin{array}{l}\text { Phosphatidylserine, Calreticulin, } \\
\text { Calnexin }\end{array}$ & Nuclear fragments and cell organelles \\
\hline
\end{tabular}

ALIX, TSG101, VPS4, heat shock protein (HSP70) and the ESCORT complex [11].

Microvesicles are formed by direct budding from the cell plasma membrane with involvement of cytoskeleton components and fusion machinery, though the process is not yet fully understood. Due to their biogenesis pathway, microvesicles are primarily composed of a plasma membrane and of cytosolic-associated proteins. Other commonly found components include heat shock proteins, integrins, posttranslationally modified proteins and RNA species. Several structural components are shared between exosomes and microvesicles due to their similar release pathways and origin [11].

EVs have been designated as novel cell-to-cell communicators due to their effect on cells on a paracrine and endocrine level, specifically through the direct stimulation of cell surface receptors and transfer of bioactive molecules. Indeed, EV surface receptors may act as signalling complexes and directly stimulate target cells or, alternatively, transfer functionally active receptors from one cell to another. For instance, bystander $\mathrm{B}$ cells can acquire antigen receptors from activated B cells becoming specific activated antigen presenting cells for CD4 T cells [12].

In addition, the presence of a complex cargo (miRNA, RNA, proteins, lipids, cytokines and mitochondria) within EVs results in a multilevel modulation of cell functions in the recipient cells $[13,14]$. Small RNA species, including miRNA, are present within EVs and, interestingly, recent studies found that the overall pattern of miRNA content of small and large EVs appears similar but distinctly different from that of the originating cells [15••], implying specific mechanisms of miRNA packaging into EVs. Moreover, extensive proteomic studies on EVs originating from cell cultures, tissue cultures and isolated bio-fluids have shown a significant EV protein content. Online databases created through the collaboration of EV research groups provide us with catalogued EV components, such as Exocarta, (www. exocarta.org), EVpedia (www.evpedia.info) and Vesiclepedia (www.microvesicles.org). EVs contain many common proteins involved in vesicle trafficking and serving as part of the cytoskeleton and the plasma membrane. Furthermore, specific protein content reflects the EV mechanism of generation and origin, as well as the cellular state of the EV originating cell. Finally, in addition to the structural functions of lipids in EV membranes, bioactive lipids such as eicosanoids, fatty acids and cholesterol are transferred by EVs to recipient cells. For instance, sphingomyelin has been shown to regulate angiogenesis in vitro and in vivo in tumour derived EVs [16].

\section{Apoptotic EVs}

Apoptosis is commonly known as programmed cell death. An apoptotic cell undergoes several morphological changes: membrane blebbing, membrane protrusion formation and generation of ApoBDs [17-19]. The membrane of ApoBDs reflects the main changes occurring in the cell surface of the apoptotic cell. In particular, apoptotic cells express markers promoting their removal by surrounding cells or macrophages before the cell membrane ruptures [20]. For instance, Calreticulin, an "eat me" ligand is physiologically silenced by the CD47 "don't eat me" ligand; and only expressed by cells and ApoBDs when CD47 is downregulated [21]. The size of ApoBDs ranges from $1 \mu \mathrm{m}$ to $5 \mu \mathrm{m}[22,23]$. This characteristic is similar to oncosomes (EVs secreted by cancer cells), but the biogenesis of these vesicles differs [24]. The number of ApoBDs produced per cell was quantified as $12.87 \pm 3.23$ per hour [25••]. In comparison, the average number of released EVs by mesenchymal stem cells was found be in the range of 2900 per cell, overnight [26].

During apoptosis, apoptotic microvesicles $0.1-1 \mu \mathrm{m}$ in diameter and small exosome-like EVs are released [27, 28]. However, these vesicles are less characterized than the ApoBDs.

ApoBDs are characterized by the presence of externalized phosphatidylserine and by a permeable membrane. As mentioned above, they express phagocytosis-promoting signals, such as calreticulin [21] and calnexin [29]. In addition, ApoBDs express chemokines and adhesion molecules, such as $\mathrm{CX} 3 \mathrm{CL} 1 /$ fractalkine and ICAM3, and MHC class II 
molecules, allowing for direct antigen presentation to $\mathrm{CD} 4^{+} \mathrm{T}$ cells and activation of immunological memory [30]. The cargo of ApoBDs consists of cellular components enclosed during protrusion. Due to this fact, the content of ApoEVs can be very diverse. Indeed, ApoBDs can contain microRNAs, RNA and DNA. Diversity of ApoBDs content affects their physiological properties. ApoBDs can be subdivided into two groups: DNA-carrying ApoBDs and cytoplasm-carrying ApoBDs. 5' phosphorylated blunt-ended DNA can be used as a distinctive marker of DNA-carrying ApoBDs because it is exclusively found in ApoBDs, which undergo apoptosis and contain the DNA fragments [31].

\section{Mitochondria}

While mitochondria are widely considered the powerhouse of the cell, as they are responsible for ATP production through oxidative phosphorylation, these organelles are also involved in several other pathways. They serve a role in pluripotent stem cell maintenance [32], apoptosis and cell death regulation and proliferation capacity through complex interactions between $\mathrm{p} 53$ and reactive oxygen species (ROS) production [32]. Energy deprivation and mitochondria dysfunction has been strictly associated with end stage organ disease [33]. Therefore, while metabolic patterns and mitochondria contents can strictly vary among the organs, it appears evident that mitochondrial alterations are closely correlated with most of the clinical conditions that lead to organ failure [34, 35].

As mitochondria do not possess an efficient DNA-repair system [36], these organelles are typically recycled through mitophagy, a form of autophagy [37]. Moreover, the transfer of respiration-competent mitochondria from cell to cell emerged in the past few years [6] as a mechanism of damage repair or cell reprogramming [38]. The physiological mitochondria transfer is a biological phenomenon in which the organelle from a healthy donor cell is relocated into a stressed recipient cell, resulting in repair and survival of the damaged cell. During this process, the mitochondria's small size and plasticity allow it to be transported from donor to recipient cells through transporting mechanisms, such as tunneling nanotubes and microvesicles. The organelle is eventually incorporated into the endogenous mitochondrial network of the recipient cell that needs to be rescued, restoring the cell to its bio-energetic profile and health [39].

The incorporation of respiration-competent mitochondria within released microvesicles has been extensively studied in MSCs, where, once internalized, mitochondria-containing microvesicles can rescue cells from injury or act as reprogramming factors $[5,40 \bullet \cdot$. In 2012, Islam et al. demonstrated that mitochondria can be transferred in vivo through a mouse model of acute lung injury [38]. Firstly, mitochondrialabelled MSCs were administered by injection into the damaged lungs. These MSCs homed in the damaged tissue and produced microvesicles containing the labelled mitochondria $4 \mathrm{~h}$ post-injection. The microvesicles were then directly transferred into the damage lung cells, resulting in the restoration of their ATP concentrations and secretory responses [38].

PMT has also been observed through the establishment of tunnelling nanotubes (TNTs) [6]. TNTs are filamentous connections formed by protrusions of a cell's membrane and are used to share organelles and contents of the cell's cytoplasm with other cells [41]. The utilization of TNTs in PMT has been observed in transfers between MSCs and macrophages, which served to enhance macrocytosis and activate antimicrobial response [42]. Moreover, Sinclair et al. demonstrated that the transfer of mitochondria via TNTs is essential due to the regenerative capacity exerted by MSCs [41]. However, the role and mechanism of PMT through the establishment of TNTs is poorly understood. The last and least known mechanism of PMT is the direct release of mitochondria in the extracellular microenvironment, usually in response to cell stress. "Naked" mitochondria can be encapsulated in a vacuole and then extruded by apoptotic cells, specifically hepatocytes [43]. Similarly, platelets can release respiration-competent mitochondria as a mediator of innate immune response [44].

\section{Therapeutic Effects of EVs, ApoBDs and Mitochondria for Organ Regeneration}

\section{EV Reprogramming of Injured Tissue}

Following the emerging interest of stem cell therapy, an increasing number of research and pharmaceutical groups are focusing on stem cell derived EVs, specifically MSC-derived EVs, as a new form of therapeutic agents for organ regeneration and protection [45-48]. Due to the ability of MSCderived EVs to transfer therapeutic molecules, such as mRNAs, miRNAs and protein, and several of their regenerating effects to MSCs, this source of EVs is one of the most studied in Regenerative Medicine. Moreover, MSCs produce a higher number of EVs in comparison with other stem cells [49]. MSC-derived EVs were proven to modulate the immune system and stimulate regeneration in a multitude of preclinical models, including graft-versus-host-disease, lung, liver, kidney and cardiovascular injury [45]. There are a significant number of studies describing the proregenerative effects of stem cell-EVs for organ regeneration, but it is not within the scope of this review to detail all the different preclinical models of application.

As detailed above, the therapeutic effect of stem cell-EVs on organ repair is related to transfer of pro-regenerative proteins or microRNAs. For instance, although numerous factors have demonstrated the therapeutic effects of different EV models and origins, not a single agent emerged as pivotal or 
indispensable. Therefore, it can be hypothesized that not a single factor, but rather a synergic and multi-target action of EV components is responsible for the therapeutic EV results. Indeed, the common and characterizing action of therapeutic MSC-EVs can be described as a reprogramming activity on tissue expression profiles. For instance, in models of kidney and liver injury [26], the expression profile of the EV-treated diseased organ, as assessed by RNA sequencing, correlated with that of the normal tissue. Moreover, in models of chronic tissue injury, an upregulation of anti-fibrotic genes and downregulation of pro-fibrotic genes was common to the different diseases as well as stem cell sources $[50,51]$. Through modulation of their phenotype and subsequent secretomes, therapeutic utilization of EVs may benefit from an in vitro stimulation or manipulation of the generating cell source. For instance, ischemic or hormonal stimulation may ameliorate EV activities [52-54]. In addition, EV administration for chronic diseases might require multiple administrations. The possible development of immune reactions in this setting has not yet been studied in depth.

\section{Regenerative Effect of Apoptotic Body Phagocytosis}

Due to the rapid clearance of damaged cells by immune cells, namely phagocytes, ApoBDs play a major role in immune regulation $[30,55]$. ApoBDs are emerging as a pivotal tool in cellto-cell communication between damaged and healthy cells, therefore modulating mechanisms of organ repair. Indeed, ApoBDs may stimulate proliferation of resident stem/progenitor cells, improving tissue regeneration and replacing damaged cells $[25 \bullet \bullet, 56]$. For instance, phagocytosis of the ApoBDs by hepatic stellate cells can promote their differentiation and increase their cell survival [57]. Moreover, ApoBDs' engulfment may support MSC homeostasis. In particular, systemic infusion of exogenous ApoBDs was able to rescue apoptotic MSCs by transferring RNF146 and miR-328-3p and activating the $\mathrm{Wnt} / \beta$-catenin signalling [58•]. In parallel, in zebrafish, dying epithelial stromal cells of the epidermis were observed to generate Wnt8a enriched ApoBDs, supporting the hypothesis of ApoBDs being biologically active vehicles in cell-to-cell communication [25••]. Neighbouring p63-positive stromal cells engulfed the ApoBDs, which caspasedependently activated Wnt signalling and stimulated cell proliferation and tissue homeostasis over $24 \mathrm{~h}$. In this model, inhibition of apoptosis significantly reduced the number of proliferating stromal cells. On the contrary, overexpression of the Wnt pathway in combination with apoptosis induced a significant increase of stromal cell proliferation [25••].

ApoBDs can deliver microRNAs, DNA and other genetic material to target cells, resulting in a multitude of different effects. For example, miRNA-126, present in endothelial ApoBDs, promoted chemokine CXCL12 expression in healthy endothelium, and repeated administration of those
ApoBDs in mice with atherosclerosis induced an atheroprotective effect [46].

Although the use of ApoBDs generated in culture as therapeutic has not yet been tested, their role appears of increasing interest in the field of Regenerative Medicine.

\section{Artificial Mitochondria Transfer}

In recent years, artificial mitochondrial transfer (AMT) emerged as a new possible therapeutic option for tissue repair. AMT has been intensively investigated in cardiac disease models. In a rabbit model of cardiac IRI, the injection of viable respiration-competent mitochondria, isolated from donor rabbit cardiac or muscular tissues, was able to significantly reduce the infarct size area, kinase MB, cardiac troponin-I and apoptosis in the regional ischemic zone [47, 48]. AMT has also been recently tested in a mouse model of heterotopic heart transplantation: mitochondria isolated from gastrocnemius muscle were autologously administrated in the heart coronary ostium before and after the transplant. Within $24 \mathrm{~h}$ after transplant, necrosis and neutrophil infiltration were significantly decreased compared with the vehicle-treated group. Moreover, the mitochondrial treatment significantly enhanced the beating score after transplant [59]. Interestingly, these papers demonstrated both in vitro and in vivo that fully differentiated cells can be used as a source for the mitochondrial injection.

Moreover, transfer of mitochondria, mainly derived from MSCs, has been proven effective in other pathologic models involving liver, brain and kidney. In a rat model of liver IRI, the intra-splenic administration of MSC mitochondria mitigated the necrosis of hepatocytes as well as reduced the expression of mitochondrial-induced apoptosis markers [60]. In the kidney, the effectiveness of AMT was demonstrated by rescue of damaged renal proximal tubular cells. In vitro, the administration of MSC-derived mitochondria reduced ROS production and increased the expression of the tubular marker megalin and mitochondrial superoxide dismutase 2, whereas in vivo, both the tubular basement membrane and brush border were protected [61]. Moreover, in normal mice, the administration of mitochondria improved endurance during forced swimming test. Finally, AMT has also been used in vivo in a murine model of Parkinson's disease and in vitro for the regeneration of damaged hippocampal cells [62, 63].

Although of great novelty, these therapeutic approaches have already started to be applied in the human setting.

\section{Clinical Trials Involving EVs and Mitochondria Transfer}

All the clinical trials concerning MSC-EVs and ATM can be found at www.clinicaltrails.gov. Although the majority of listed trials focus on the diagnostic properties of EVs, there 
are five trials testing the therapeutic applications of MSCsEVs and two proposing the use of ATM (Table 2).

A first trial is designed to test the anti-inflammatory properties of umbilical cord derived MSCs-EVs to prevent the destruction of pancreatic $\beta$-cell islets. The MSCs-EVs will be administered intravenously in two doses, the first dose of exosomes and, after seven days, the second dose of microvesicles (NCT02138331). Two other clinical trials using EVs will involve allogeneic MSCs-EVs. One of them will administer EVs enriched by miR-124 for the treatment of acute ischemic stroke (NCT03384433). The second clinical trial will attempt to treat lesions in patients affected by dystrophic epidermolysis bullosa (NCT04173650). The last clinical trial using EVs that is in the recruiting phase focuses on promoting the healing and recovery of refractory macular holes through direct injection of MSC exosomes to the site of the injury (NCT03437759). Finally, the only concluded trail to date used umbilical cord derived MSCs-EVs to inhibit the progression of chronic kidney disease in patients with grade III-IV CKD [64]. The study showed stabilization of the disease progression, as confirmed by stable levels of glomerular filtration rate, serum creatinine and blood urea in treated patients, and an increased level of anti-inflammatory factors (TGF- $\beta 1$ and IL-10) in comparison with the matching placebo group.

The first clinical trial using administration of isolated mitochondria for the treatment of myocardial IRI has also been concluded with positive results [65]. Mitochondria were isolated from non-ischemic skeletal muscles and injected in the myocardium of paediatric patients with myocardial IRI. No adverse effects were detected after AMT, and four out of five patients demonstrated an enhancement in ventricular function [65].

Other clinical trials using AMT are focused on the improvement of infertility treatments (Table 2). Through autologous micro-injection of mitochondria prior to intracytoplasmic sperm injection, the patients' oocyte quality was enhanced. In the first trial, concluded in 2017, mitochondria were isolated from autologous ovarian stem cells and directly injected into the oocytes themselves. To date, no results have been published. Embryo quality has been quantified through the pregnancy rate after treatment and morphological evaluation of the treated embryos. In the second clinical trial, which is still ongoing, mitochondria will be isolated from autologous bone marrow-MSCs and administrated immediately before intra-cytoplasmic sperm injection in the oocytes. Live birth

Table 2 Current clinical trials concerning the use of EVs or AMT as therapeutic agents

\begin{tabular}{|c|c|c|c|c|c|c|}
\hline & Intervention & N. pts & Follow up & State & Location & Number/Ref. \\
\hline Diabetes Mellitus type 1 & $\begin{array}{l}\text { Two doses of } \\
\text { MSC-EVs }\end{array}$ & 20 & 3 months & Unknown & $\begin{array}{l}\text { Sahel Teaching } \\
\text { Hospital Sahel, } \\
\text { Cairo, Egypt }\end{array}$ & NCT02138331 \\
\hline $\begin{array}{l}\text { Chronic kidney } \\
\text { disease }\end{array}$ & $\begin{array}{l}\text { Two doses of } \\
\text { umbilical cord } \\
\text { MSC-EVs } \\
(100 \mu \mathrm{g} / \mathrm{kg} / \text { dose })\end{array}$ & 20 & 1 year & Concluded & $\begin{array}{l}\text { Sahel Teaching } \\
\text { Hospital Sahel, } \\
\text { Cairo, Egypt }\end{array}$ & Nassar et al. \\
\hline $\begin{array}{l}\text { Molecular } \\
\text { degeneration }\end{array}$ & $\begin{array}{l}20-50 \mathrm{mg} \text { of cord } \\
\text { tissue MSC-EVs } \\
\text { injected directly } \\
\text { around macular hole }\end{array}$ & 44 & 24 weeks & Recruiting & $\begin{array}{l}\text { Tianjin Medical } \\
\text { University } \\
\text { Hospital Tianjin, } \\
\text { China }\end{array}$ & NCT03437759 \\
\hline $\begin{array}{l}\text { Cerebrovascular } \\
\text { disorders acute } \\
\text { ischemic stroke }\end{array}$ & $\begin{array}{l}\text { Allogeneic MSC-EVs } \\
\text { enriched by miR-124 }\end{array}$ & 5 & 12 months & Not recruiting yet & $\begin{array}{l}\text { Shahid Beheshti } \\
\text { University } \\
\text { of Medical } \\
\text { Sciences, Tehran, } \\
\text { Iran }\end{array}$ & NCT03384433 \\
\hline Dystrophic Epidermolysis Bullosa & $\begin{array}{l}\text { Allogeneic MSC-EVs } \\
\text { applied directly to } \\
\text { lesions, blisters for } \\
60 \text { days }\end{array}$ & 30 & 2 months & Not recruiting yet & $\begin{array}{l}\text { Aegle Therapeutics, } \\
\text { Miami, Florida } \\
\text { USA }\end{array}$ & NCT04173650 \\
\hline Repetition failure & $\begin{array}{l}\text { Clinical Application of } \\
\text { Autologous } \\
\text { Mitochondria Transfer } \\
\text { for Improving } \\
\text { Oocyte Quality. }\end{array}$ & 50 & $2-3$ years & Not recruiting yet & & NCT03639506 \\
\hline Infertility & $\begin{array}{l}\text { Amelioration of oocyte } \\
\text { quality using autologous } \\
\text { mitochondria transfer }\end{array}$ & 59 & 5 months & Concluded & Valencia, Spain & NCT02586298 \\
\hline IRI & $\begin{array}{l}\text { Autologous mitochondrial } \\
\text { transfer for dysfunction } \\
\text { after ischemia-reperfusion injury }\end{array}$ & 5 & 6 days & Concluded & Boston, MA, USA & Emani et al. \\
\hline
\end{tabular}


rate, pregnancy rate, number of oocytes retrieved and fertility rate are going to be evaluated.

\section{Conclusion}

Numerous discoveries within the Regenerative Medicine field have highlighted the bioactivity of stem cell bio-products and their role in cell-to-cell communication. In particular, EVs are the most advanced as potential therapeutic agents due to their ability to modulate the function of targeted cells. Together with stem cell-EVs, proven to be of therapeutic effect in a large variety of pre-clinical models, ApoBDs and AMT can be utilized for selected and specific applications.

The major issue with use of EVs in clinical practice is the standardization of EVs isolation, usage and storage. However, once those issues can be overcome, using EVs as therapeutic agents provides solutions to numerous complications caused by stem-cell therapy, including immune compatibility, maldifferentiation and tumourigenicity. EV therapy allows for dosage control, evaluation of safety and potency equivalent to pharmaceutical agents. In comparison to stem cell therapy, EVs are potentially an easier option as they can be directly obtained from the medium of cultured cells, massively produced and stored without the application of toxic cryopreservative agents and/or loss of EV potency [66]. The biological properties of $\mathrm{EV}$ allow for modification of the EV content to obtain desired cell-specific effects. Encapsulation of effector molecules (nucleic acids, lipids and proteins) by EVs allows delivery of its cargo without the risk of degradation. Genetically modified EVs may offer a more effective and natural solution than usage of FDA-approved lipid nanoparticles, which pose a low-dose toxicity upon cell entry [67].

The application of ApoBDs is still poorly explored, as several aspects still require further investigation. For instance, apoptotic vesicles are quite heterogeneous and may have different compositions and properties depending on their size. While EVs released by primary murine aortic endothelial apoptotic cells enhanced inflammation in mice transplanted with an MHC-incompatible graft, ApoBDs did not show this behaviour [28]. Therefore, as increasing experimental evidence suggests, the therapeutic effects of stem cells are due to their clearance by the immune system [4, 68]. For this reason, therapeutic utilization of these cell products appears of interest.

Finally, the use of MSCs as a source of mitochondria for AMT is a novel, therapeutic option that shows regenerative effects in the treatment of acute cell damage. However, more studies are required for better understanding of mitochondria internalization, their fate once inside the injured cells and how mitochondria can survive in the extracellular microenvironment or the blood flow during AMT.
Acknowledgements We would like to thank Lola Buono for her contribution to the review by designing Figure 1 .

Funding This project has received funding from the European Union's Horizon 2020 research and innovation programme under the Marie Sklodowska-Curie grant agreements No. 813839 and 765274.

\section{Compliance with Ethical Standards}

Conflict of Interest All authors declare no conflict of interest.

Human and Animal Rights and Informed Consent This article does not contain any studies with human or animal subjects performed by any of the authors.

Open Access This article is licensed under a Creative Commons Attribution 4.0 International License, which permits use, sharing, adaptation, distribution and reproduction in any medium or format, as long as you give appropriate credit to the original author(s) and the source, provide a link to the Creative Commons licence, and indicate if changes were made. The images or other third party material in this article are included in the article's Creative Commons licence, unless indicated otherwise in a credit line to the material. If material is not included in the article's Creative Commons licence and your intended use is not permitted by statutory regulation or exceeds the permitted use, you will need to obtain permission directly from the copyright holder. To view a copy of this licence, visit http://creativecommons.org/licenses/by/4.0/.

\section{References}

Papers of particular interest, published recently, have been highlighted as:

- Of importance

•- Of major importance

1 Roesnbloom J, Castro SV, Jimenez SA. Review narrative review: fibrotic diseases: cellular and molecular mechanisms and novel therapies. Ann Intern Med. 2010;152(3):159-66.

2 Jha $\mathrm{V}$, et al. Chronic kidney disease: Global dimension and perspectives. Lancet. 2013;382(9888):260-72.

3 Chawla LS, Eggers PW, Star RA, Kimmel PL. Acute kidney injury and chronic kidney disease as interconnected syndromes. N Engl J Med. 2014;371(1):58-66.

4 Galleu A, et al. Apoptosis in mesenchymal stromal cells induces in vivo recipient-mediated immunomodulation. Sci Transl Med. 2017:9:416.

5 Islam MN, et al. Mitochondrial transfer from bone-marrow-derived stromal cells to pulmonary alveoli protects against acute lung injury. Nat Med. 2012;18(5):759-65.

6 Caicedo A, Aponte PM, Cabrera F, Hidalgo C, Khoury M. Artificial mitochondria transfer: current challenges, advances, and future applications. Stem Cells Int. 2017;2017:7610414.

7 Murray LMA, Krasnodembskaya AD. Concise review: intercellular communication via organelle transfer in the biology and therapeutic applications of stem cells. Stem Cells. 2019;37(1):14-25.

8 Chargaff $\mathrm{E}$, West R. The biological significance of the thromboplastic protein of blood. 1946;166(1):189-97.

9•• Théry C, et al. Minimal information for studies of extracellular vesicles 2018 (MISEV2018): a position statement of the International Society for Extracellular Vesicles and update of the MISEV2014 
guidelines. J Extracell Vesicles. 2018;7(1). The paper provides a throughout description of minimal information needed to work with extracellular vesicles as well as their chracterisation and description.

10 Akers JC, Gonda D, Kim R, Carter BS, Chen CC. Biogenesis of extracellular vesicles (EV): Exosomes, microvesicles, retroviruslike vesicles, and apoptotic bodies. J Neurooncol. 2013;113(1):111.

11 Buzas EI, et al. Biological properties of extracellular vesicles and their physiological functions. 2015;1:1-60.

12 Quah BJC, Barlow VP, McPhun V, Matthaei KI, Hulett MD, Parish CR. Bystander B cells rapidly acquire antigen receptors from activated $B$ cells by membrane transfer. Proc Natl Acad Sci U S A. 2008;105(11):4259-64.

13 Chai R, et al. Exosome secreted by MSC reduces myocardial ischemia/reperfusion injury. Stem Cell Res. 2010;4(3):214-22.

14 Mathivanan S, Ji H, Simpson RJ. Exosomes: extracellular organelles important in intercellular communication. J Proteomics. 2010;73(10):1907-20.

15•- Jeppesen DK, et al. Reassessment of Exosome Composition. Cell. 2019;177(2):428-445.e18. The recent study shows the composisiton of exosomes to be different than prviously thought, thus changing the ideas behing exosomes and their potential applications.

16 Kim CW, Lee HM, Lee TH, Kang C, Kleinman HK, Gho YS. Extracellular membrane vesicles from tumor cells promote angiogenesis via sphingomyelin. Cancer Res. 2002;62(21):6312-7.

17 Fadeel B, Orrenius S. Apoptosis: a basic biological phenomenon with wide-ranging implications in human disease. Br J Cancer. 1972;26:239-57.

18 Atkin-Smith GK, et al. A novel mechanism of generating extracellular vesicles during apoptosis via a beads-on-a-string membrane structure. Nat Commun. 2015;6:7439.

19 Alberts B, Bray D, Alexander J, Lewis J. Základy buněčné biologie. Espero: Partizánske; 2006.

20 Depraetere V. 'Eat me' signals of apoptotic bodies. Nat Cell Biol. 2000;2(6):2000.

21 Gardai SJ, et al. Cell-surface calreticulin initiates clearance of viable or apoptotic cells through trans-activation of LRP on the phagocyte. Cell. 2005;123(2):321-34.

22 Hristov M, Erl W, Linder S, Weber PC. Apoptotic bodies from endothelial cells enhance the number and initiate the differentiation of human endothelial progenitor cells in vitro. Blood. 2004;104(9): 2761-6.

23 Tixeira R, et al. Defining the morphologic features and products of cell disassembly during apoptosis. Apoptosis. 2017;22(3):475-7.

24 Jiang L, et al. Determining the contents and cell origins of apoptotic bodies by flow cytometry. Sci Rep. 2017;7(1):1-12.

$25 \bullet$ Brock CK, et al. Stem cell proliferation is induced by apoptotic bodies from dying cells during epithelial tissue maintenance. Nat Commun. 2019;10(1):1-11. This recent study shows that ApoBDs stimulates proliferation of stem cells improving tissue regeneration.

26 Collino F, et al. AKI recovery induced by mesenchymal stromal cell-derived extracellular vesicles carrying microRNAs. J Am Soc Nephrol. 2015;26(10):2349-60.

27 Karpman D, Ståhl AL, Arvidsson I. Extracellular vesicles in renal disease. Nat Rev Nephrol. 2017;13(9):545-62.

28 Dieudé M, et al. The 20S proteasome core, active within apoptotic exosome-like vesicles, induces autoantibody production and accelerates rejection. Sci Transl Med. 2015;7(318):1-18.

29 Lunavat TR, et al. Small RNA deep sequencing discriminates subsets of extracellular vesicles released by melanoma cells - evidence of unique microRNA cargos. RNA Biol. 2015;12(8):810-23.

30 Caruso S, Poon IKH. Apoptotic cell-derived extracellular vesicles: More than just debris. Front Immunol. 2018;9:1486.
31 Hauser P, Wang S, Didenko VV. Apoptotic Bodies: Selective Detection in Extracellular Vesicles. Methods Mol Biol. 2017;1554: $127-41$.

32 Folmes CDL, et al. Somatic oxidative bioenergetics transitions into pluripotency-dependent glycolysis to facilitate nuclear reprogramming. Cell Metab. 2011;14(2):264-71.

33 Triolo F, Gridelli B. End-stage organ failure: Will regenerative medicine keep its promise? Cell Transplant. 2006;15(SUPPL. 1):S3-10.

34 Červinka M. The role of mitochondria in apoptosis induced in vitro. Gen Physiol Biophys. 1999;18:33-40.

35 Jassem W, Heaton ND. The role of mitochondria in ischemia/ reperfusion injury in organ transplantation. Kidney Int. 2004;66(2):514-7.

36 Zinovkina LA. Mechanisms of mitochondrial DNA repair in mammals. Biochem. 2018;83(3):233-49.

37 Ding WX, Yin XM. Mitophagy: mechanisms, pathophysiological roles, and analysis. Biol Chem. 2012;393(7):547-64.

38 Sinha P, Islam MN, Bhattacharya S, Bhattacharya J. Intercellular mitochondrial transfer: bioenergetic crosstalk between cells. Curr Opin Genet Dev. 2016;38(June):97-101.

39 Pacak CA, et al. Actin-dependent mitochondrial internalization in cardiomyocytes: Evidence for rescue of mitochondrial function. Biol Open. 2015;4(5):622-6.

40•• Phinney DG, et al. Mesenchymal stem cells use extracellular vesicles to outsource mitophagy and shuttle microRNAs. Nat Commun. 2015;6:8472. In this study phinney et al. shed light on the complex mechanism that allow macrophages to engulf mitochondria from MSCs, in order to increase their bioenergetics after oxidative stress increase.

41 Sinclair KA, Yerkovich ST, Hopkins PMA, Chambers DC. Characterization of intercellular communication and mitochondrial donation by mesenchymal stromal cells derived from the human lung. Stem Cell Res Ther. 2016;7(1):1-10.

42 Jackson MV, et al. Mitochondrial transfer via tunneling nanotubes is an important mechanism by which mesenchymal stem cells enhance macrophage phagocytosis in the in vitro and in vivo models of ARDS. Stem Cells. 2016;34(8):2210-23.

43 Nakajima A, Kurihara H, Yagita H, Okumura K, Nakano H. Mitochondrial extrusion through the cytoplasmic vacuoles during cell death. J Biol Chem. 2008;283(35):24128-35.

44 Boudreau LH, et al. Platelets release mitochondria serving as substrate for bactericidal group IIA-secreted phospholipase a to promote inflammation. Blood. 2014;124(14):2173-83.

45 Mendt M, Rezvani K, Shpall E. Mesenchymal stem cell-derived exosomes for clinical use. Bone Marrow Transplant. 2019;54:789 92.

46 Zernecke A, et al. Delivery of microRNA-126 by apoptotic bodies induces CXCL12-dependent vascular protection. Sci. Signal. 2009;2(100):1-13.

47 Shi X, Zhao M, Fu C, Fu A. Intravenous administration of mitochondria for treating experimental Parkinson's disease. Mitochondrion. 2017;34:91-100.

48 Masuzawa A, et al. Transplantation of autologously derived mitochondria protects the heart from ischemia-reperfusion injury. Am. J Physiol Hear Circ Physiol. 2013;304(7):966-82.

49 Gatti S, et al. Microvesicles derived from human adult mesenchymal stem cells protect against ischaemia-reperfusion-induced acute and chronic kidney injury. Nephrol Dial Transplant. 2011;26(5):147483.

50 Bruno S, et al. HLSC-derived extracellular vesicles attenuate liver fibrosis and inflammation in a murine model of non-alcoholic steatohepatitis. Mol Ther. 2020;28(2):1-11.

51 Grange C, Skovronova R, Marabese F, Bussolati B. Stem cellderived extracellular vesicles and kidney regeneration. Cells. 2019;8(10): 1240 . 
52 Zhu J, et al. Myocardial reparative functions of exosomes from mesenchymal stem cells are enhanced by hypoxia treatment of the cells via transferring microRNA-210 in an nSMase2-dependent way. Artif Cells Nanomed Biotechnol. 2018;46(8):1659-70.

53 Lopatina T, Bruno S, Tetta C, Kalinina N, Porta M, Camussi G. Platelet-derived growth factor regulates the secretion of extracellular vesicles by adipose mesenchymal stem cells and enhances their angiogenic potential. Cell Commun Signal. 2014;12(1):1-12.

54 Zanjani ED, Banisadre M. Hormonal stimulation of erythropoietin production and erythropoiesis in anephric sheep fetuses. J Clin Invest. 1979;64(5):1181-7.

55 Caruso S, et al. Defining the role of cytoskeletal components in the formation of apoptopodia and apoptotic bodies during apoptosis. Apoptosis. 2019;24(11-12):862-77.

$56 \mathrm{Li} \mathrm{B}$, et al. Bone marrow mesenchymal stem cells protect alveolar macrophages from lipopolysaccharide-induced apoptosis partially by inhibiting the $\mathrm{Wnt} / \beta$-catenin pathway. Cell Biol Int. 2015;39(2):192-200.

57 Jiang JX, Mikami K, Venugopal S, Li Y, Török NJ. Apoptotic body engulfment by hepatic stellate cells promotes their survival by the JAK/STAT and Akt/NF-kB-dependent pathways. J Hepatol. 2009;51(1):139-48.

58 - Liu D, et al. Circulating apoptotic bodies maintain mesenchymal stem cell homeostasis and ameliorate osteopenia via transferring multiple cellular factors. Cell Res. 2018;28(9):918-33. The paper shows ApoBDs activates Wnt/ $\beta$-catenin signalling which can ameliorate MSCs in apoptosis.

59 Moskowitzova K, et al. Mitochondrial transplantation prolongs cold ischemia time in murine heart transplantation. J HearT Lung Transplant. 2019;38(1):92-9.
60 Lin HC, Liu SY, Lai HS, Lai IR. Isolated mitochondria infusion mitigates ischemia-reperfusion injury of the liver in rats. Shock. 2013;39(3):304-10.

61 Konari N, Nagaishi K, Kikuchi S, Fujimiya M. Mitochondria transfer from mesenchymal stem cells structurally and functionally repairs renal proximal tubular epithelial cells in diabetic nephropathy in vivo. Sci Rep. 2019;9(1):1-14.

62 Chien L, Liang MZ, Chang CY, Wang C, Chen L. Mitochondrial therapy promotes regeneration of injured hippocampal neurons. Biochim Biophys Acta - Mol Basis Dis. 2018;1864(9):3001-12.

63 Shi X, Zhao M, Fu C, Fu A. Intravenous administration of mitochondria for treating experimental Parkinson's disease. Mitochondrion. 2017;34:91-100.

64 Nassar W, et al. Umbilical cord mesenchymal stem cells derived extracellular vesicles can safely ameliorate the progression of chronic kidney diseases. Biomater Res. 2016;20(21):1-11.

65 Emani SM, Piekarski BL, Harrild D, del Nido PJ, McCully JD. Autologous mitochondrial transplantation for dysfunction after ischemia-reperfusion injury. J Thorac Cardiovasc Surg. 2017;154(1):286-9.

66 Ohno SI, Drummen GPC, Kuroda M. Focus on extracellular vesicles: Development of extracellular vesicle-based therapeutic systems. Int J Mol Sci. 2016;17(2):172.

67 Murphy DE, et al. Extracellular vesicle-based therapeutics : natural versus engineered targeting and trafficking. Exp Mol Med. 2019;51: $1-12$.

68 Vagnozzi RJ, et al. An acute immune response underlies the benefit of cardiac stem-cell therapy. Nature. 2019;577:405-9.

Publisher's Note Springer Nature remains neutral with regard to jurisdictional claims in published maps and institutional affiliations. 